\title{
Descripción de casos de absceso pectoral crónico y análisis de algunas variables asociadas a su presentación en equinos de la Provincia de Nuble, Chile ${ }^{\#}$
}

\author{
Case descriptions of chronic pectoral abscess and variables associated with its occurrence in \\ equines, in the Nuble Province, Chile \\ P Gädicke ${ }^{a *}$, G Azócar ${ }^{b}$, M Ocaña ${ }^{a}$ \\ aDepartamento de Patología y Medicina Preventiva, Facultad Medicina Veterinaria, Universidad de Concepción, Chillán, Chile.

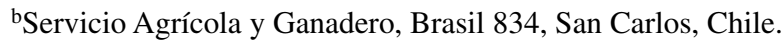

\begin{abstract}
SUMMARY
The chronic pectoral abscess (APC) is a seasonal infectious disease which affects equines and it is caused by Corynebacterium pseudotuberculosis. A cross-sectional study was carried out in 31 herds of the Nuble Province, VIII Region, Chile, that had reported cases of APC from November of 1999 to April of 2000 to the National Animal Health Service (SAG). Individual information and handling of all the equines from herds of reported cases, was collected by means of a survey developed by SAG. The most frequent signs were: external abscesses (79.6\%), oedema (100\%), decay (61.1\%) and fever $(31.5 \%)$. Necrosis was not reported. $83.3 \%$ of the affected equines recovered within three weeks, whereas $12.9 \%$ developed a chronic condition; mortality was $1.8 \%$. A final model was developed by means of a multiple logistic regression analysis, using Stata S/E V9 (StataCorp 2005). The model indicated that the factors associated with an increase of the probability of disease were the horses being of the Creole type $(\mathrm{OR}=6.17$; IC 95\% $=2.43-15.69)$ and administrating antiparasitic once a year $(\mathrm{OR}=4.8 ; \mathrm{IC} 95 \%=2.13-10.79)$ with a prediction level of $70.5 \%$. It is necessary to study the intrinsic and extrinsic characteristics of creole equines that make them more susceptible to APC. The relationship between the disease and the characteristics of the used annual antiparasitic should be studied to determine the link between them. Further studies consisting of observational designs would allow the analysis of causality which would help in the design of a causal model for this disease in the VIII Region.
\end{abstract}

Palabras clave: absceso Corynebacterium pseudotuberculosis, equinos.

Key words: abscess, Corynebacterium pseudotuberculosis, equine.

\section{INTRODUCCION}

En el año 1959, en caballos del estado de California (EE.UU.) se describió un cuadro durante los meses de calor, el cual se presentó como absceso pectoral y abdominal crónico (APC) desde el cual se aisló e identificó como agente causal Corynebacterium pseudotuberculosis (Wisecup y col 1964), el cual es un parásito intracelular facultativo que en el ambiente se encuentra principalmente en el suelo y en el estiércol contaminado con exudados purulentos (Burrell 1981). En la mayoría de los casos se describe que los abscesos ocurren en los músculos del área pectoral. Ocasionalmente también se encuentran en la región abdominal ventral, desde el ombligo hasta la glándula mamaria o zona prepucial (Hughes y Biberstein 1959). La mayoría de los caballos no demuestra manifestaciones sistémicas de la enfermedad; sin embargo, en algunos casos se presenta fiebre, depresión, inapetencia, cojeras y dermatitis de la línea media (Colahan y col 1991). En algunos caballos se presentan, además de los abscesos superficiales, un gran

\footnotetext{
Aceptado: 08.05.2007.

\# Financiado por: Servicio Agrícola y Ganadero, oficina San Carlos.

* Picarte 3675, Valdivia, Chile; pgadicke@udec.cl
}

número de abscesos internos los cuales pueden afectar el peritoneo, mesenterio, hígado, bazo, riñones, útero, diafragma, pulmones y pleura (Rumbaugh y col 1978), con la consecuente pérdida de peso, cólico o la ataxia que éstos pueden causar (Fraser y col 1993). Aunque los abscesos internos son menos comunes que los abscesos externos (Aleman y col 1996), se describen casos de pericarditis y pleuritis causadas por Corynebacterium pseudotuberculosis (Perkins 2004). Se ha estudiado su participación como una de las causas de la sarcoidosis equina, pero no se ha logrado demostrar que lo sea (Spiegel y col 2006). La tasa de mortalidad por este agente es muy baja, generalmente se asocia a abscesos internos, animales debilitados o a tratamientos tardíos (Colahan y col 1991).

La frecuencia de aparición de casos de APC aumenta generalmente en los meses de calor de años de alta pluviosidad (Pinochet 1992). Como puerta de entrada del agente se sospecha las escoriaciones y grietas en el tegumento; sin embargo, debido al aumento estacional se ha sugerido su transmisión por insectos vectores (Blood y Radostits 1992, Doherr y col 1999). En estudios retrospectivos, se ha determinado que la incidencia aumenta luego de inviernos muy lluviosos, los que mejorarían las condiciones para el desarrollo de insectos en el verano y otoño siguientes (Miers y Ley 1980). La incidencia de la presentación de APC también varía considerablemente 
entre los años (Aleman y col 1996); una vez que se presenta la enfermedad en una determinada región, podrían continuar apareciendo casos esporádicamente durante los años siguientes (Doherr y col 1999).

No se reporta que la edad sea un factor predisponente en esta enfermedad, sin embargo la infección raramente se encuentra en animales menores de un año (Aleman y col 1996). En un estudio retrospectivo que consideró 117 caballos se determinó un rango de edad de 1 mes a 21 años (Miers y Ley 1980). La media de edad de los caballos enfermos con abscesos externos fue de 5 años, sugiriendo tal vez que los caballos jóvenes tienen mayor riesgo de infección, sin embargo, los potrillos menores de seis meses presentan una baja incidencia de infección, pudiendo ser protegidos por inmunidad materna (Aleman y col 1996). Los primeros estudios realizados plantean que no existiría predisposición por raza, sexo y tipo de trabajo del animal (Knight 1969), lo que corrobora posteriormente Aleman y col (1996). En brotes donde se determinaron las características moleculares de Corynebacterium pseudotuberculosis, no se encontró una fuente común de infección; los autores señalan posibles factores del huésped o ambientales que faciliten la infección (Foley 2004).

Cuando se presentan casos en un predio se recomienda el aislamiento de los animales afectados, control de moscas, mantener un buen estado sanitario de los animales, desinfección de fomites contaminados y una cuidadosa disposición de las camas en las pesebreras (Aleman y col 1996); además, la remoción frecuente de la descarga de los abscesos y protección de heridas en casos de dermatitis ventral de la línea media (Miers y Ley 1980).

El año 1998 durante los meses estivales se denunciaron al Servicio Agrícola y Ganadero (SAG) de la Provincia de Nuble, gran cantidad de casos de equinos con inflamación principalmente en el área pectoral, glándula mamaria o prepucio, con la siguiente abscedación de algunas de ellas; desde las lesiones se aisló Corynebacterium pseudotuberculosis. Al año siguiente durante la misma época se recibieron denuncias de aparición de nuevos casos en la oficina del (SAG) de Chillán. Por esta razón este Servicio realizó una investigación epidemiológica, planteando la hipótesis de que existen factores individuales de los animales (edad, categoría, condición corporal) y ambientales (uso que se le da a los equinos, origen del agua de consumo, origen, uso de antiparasitarios, presencia de Haematobia irritans) que son potenciales factores de riesgo para la presentación de APC.

En el presente estudio se analiza la asociación de algunos factores con el brote de casos de APC en equinos de la Provincia de Ñuble (VIII Región de Chile) en la temporada estival 1999-2000.

\section{MATERIAL Y METODOS}

Se realizó un estudio de tipo transversal en 31 predios de la Provincia de Nuble que denunciaron casos de APC al
SAG, desde noviembre de 1999 hasta abril de 2000. Los casos de APC fueron confirmados por un Médico Veterinario del SAG oficina Chillán, luego de la denuncia presentada por el propietario o su Médico Veterinario asesor.

Se recolectó información de todos los equinos de los predios que reportaron casos, hayan enfermado o no, mediante un cuestionario que fue elaborado por el SAG para esta ocasión, considerando los siguientes aspectos: antecedentes del animal como sexo, edad (en escala continua), raza, categoría (potro, yegua, cría, caballo), estado corporal (bueno, regular), tipo de estabulación (permanente, temporal o ninguna), utilización del equino (tiro, paseo, deporte, sin amansar), origen del agua de consumo (canal, pozo), procedencia (mismo predio, otro predios, feria), alimentación (sólo pastoreo, mixto), presencia de moscas de los cuernos en el predio (sí/no), frecuencia del uso de antiparasitarios (anual, ocasional, nunca). El diagnóstico y la calificación de caso, fueron realizados por un Médico Veterinario, se registraron los signos clínicos, el tratamiento antibiótico aplicado, el tiempo de recuperación, o si la lesión se tornó crónica o el animal murió.

Análisis estadístico. La información de los cuestionarios fue consolidada mediante el programa Access 2.0 ®. Se aplicó estadística descriptiva (frecuencias absolutas y relativas) para caracterizar la presentación de la enfermedad, la evolución de los casos y las características de todos los equinos presentes en los predios.

Para evaluar qué características de los equinos pudiesen estar asociadas a la presentación de APC, se aplicó un modelo de regresión logística múltiple, utilizando Stata S/E V9 (StataCorp 2005), considerando como variable dependiente de tipo dicotómica la presentación de APC, mientras que las características individuales y de manejo de los equinos fueron las variables independientes. El modelo tuvo la siguiente estructura (Dohoo y col 2003):

$$
\ln \left[\frac{p}{1-p}\right]=B_{0}+\sum B_{j} X_{j}
$$

En donde:

$\mathrm{p}=$ probabilidad de que ocurra un caso de APC (variable dependiente)

$\beta_{j}=$ coeficiente de regresión que indica el efecto de la variable $\mathrm{X}_{j}$ sobre la probabilidad que ocurra la variable dependiente modelada.

$\beta_{0}=$ intercepto de la regresión

Para construir el modelo que mejor se ajustó a los datos, se utilizó el enfoque descrito por (Dohoo y col 2003). Se calcularon las Odds Ratio (OR) en forma univariada, mediante regresiones logísticas simples. Se identificaron las variables confusoras mediante la evaluación de la magnitud del cambio que produce en los OR de las otras variables independientes, al comparar un modelo con la variable analizada y otro sin ella, si el cambio en algún OR es importante (mayor al 15\%), indica que esta 
variable está asociada tanto con la variable dependiente, como con alguna variable independiente. La elección de las variables que se incluyen en el modelo se realizó mediante la metodología denominada eliminación hacia atrás "stepwise backward", la decisión de inclusión se tomó basada en el resultado de la prueba de razones de máxima verosimilitud (Likelihood ratio test (LRT)) con un valor de significancia de $\mathrm{P}<0,05$, cabe destacar que los factores que anteriormente hayan sido identificados como confusores no deben ser eliminados del modelo. En cuanto a la interpretación de los indicadores, se consideraron como variables estadísticamente asociadas a la presentación de APC aquellas que presentaron un valor de $\mathrm{P}<0,05$ para la prueba de Wald y que en el Intervalo de Confianza 95\% (IC95\%) del OR no incluyeron el valor 1. La evaluación de la habilidad predictiva del modelo elegido se hizo mediante el cálculo de su sensibilidad, especificidad y valores predictivos.

\section{RESULTADOS}

Se aplicó el cuestionario en 31 predios que presentaron casos de equinos con APC desde noviembre de 1999 hasta abril de 2000, en los que se registraron datos de 139 animales, de los cuales $54(38,8 \%)$ desarrollaron la enfermedad; de ellos $70 \%$ fueron hembras y $30 \%$ machos. Los signos más frecuentes consistieron en abscesos externos $(79,6 \%)$, edema en el $100 \%$ de los casos, siendo las ubicaciones más reiteradas la pectoral $(64,8 \%)$ y ventral $(25,9 \%)$; sin embargo, no se presentó necrosis, hubo decaimiento en el $61,1 \%$ de los casos y fiebre en el 31,5\% (cuadro 1).

Recibieron tratamiento antibiótico 48 animales $(88,8 \%)$ de los cuales se recuperó el 87,5\%; de ellos el 83,3\% lo hizo a las 3 semanas, mientras que el 12,9\% siguió un curso crónico. La letalidad fue del 1,8\% (cuadro 2).

La edad de los animales enfermos fluctuó entre 1 y 20 años, y en los no enfermos entre 0,2 y 23 años, mientras que la media y error estándar (SE) en los enfermos fue de $7,5(1,0)$ años y en los no enfermos 7,5 $(0,8)$ años.

Los antecedentes recolectados de los animales se indican en el cuadro 3. La categoría yegua fue la más frecuente $(64,0 \%)$. Los equinos presentaban buen estado corporal $(85,6 \%)$, la mayoría de ellos no estaba estabulada $(81,3 \%)$. La mayor utilidad que se le daba a los animales era para tiro (45,3\%), seguido de paseo (23,7\%). El 69,0\% consumía agua de canal y el 30,9\% de pozo. La mayoría de los equinos presentes en los predios, en el período del brote, era procedente del mismo predio $(88,5 \%)$, seguida de procedencia de otros predios $(7,2 \%)$. En cuanto al tipo genético de animal, el 66,9\% era de tipo criollo y el 33,1\% de raza. Mayoritariamente los animales se alimentaban mediante pastoreo directo $(75,5 \%)$. El uso de antiparasitario en forma anual fue de $48,2 \%$, ocasional de $36,0 \%$, mientras que el $15,8 \%$ de los equinos no recibía antiparasitarios. Todos los equinos al momento de ocurrir
Cuadro 1. Frecuencia absoluta y relativa (\%) de los signos en los casos de APC.

cases of APC

Absolute and relative frequency $(\%)$ of the signs in the

\begin{tabular}{|c|c|c|}
\hline \multirow{2}{*}{ Signología } & \multicolumn{2}{|c|}{ Frecuencia } \\
\hline & $\mathrm{N}^{\circ}$ & $\%$ \\
\hline \multicolumn{3}{|l|}{ Abscesos externos } \\
\hline Sí & 43 & 79,6 \\
\hline No & 11 & 20,3 \\
\hline \multicolumn{3}{|l|}{ Edema } \\
\hline Sí & 54 & 100 \\
\hline No & 0 & 0 \\
\hline \multicolumn{3}{|l|}{ Ubicación del edema } \\
\hline Ventral & 14 & 25,9 \\
\hline Pecho & 35 & 64,8 \\
\hline Miembro anterior & 3 & 5,6 \\
\hline Miembro posterior & 2 & 3,7 \\
\hline \multicolumn{3}{|l|}{ Necrosis } \\
\hline Sí & 0 & 0 \\
\hline No & 54 & 100 \\
\hline \multicolumn{3}{|l|}{ Decaimiento } \\
\hline Sí & 33 & 61,1 \\
\hline No & 21 & 38,8 \\
\hline \multicolumn{3}{|l|}{ Fiebre } \\
\hline Sí & 17 & 31,5 \\
\hline No & 37 & 68,5 \\
\hline
\end{tabular}

Cuadro 2. Frecuencia absoluta y relativa (\%) de la evolución de los casos de APC.

cases of APC.

Absolute and relative frequency (\%) of the evolution of the

\begin{tabular}{lrc}
\hline \multirow{2}{*}{$\begin{array}{c}\text { Evolución de los casos } \\
\text { de APC }\end{array}$} & \multicolumn{2}{c}{ Frecuencia } \\
\cline { 2 - 3 } & $\mathrm{N}^{\circ}$ & $\%$ \\
\hline Total Recuperados & 46 & 83,3 \\
1 semana & 9 & 16,6 \\
2 semanas & 22 & 40,7 \\
3 semanas & 15 & 27,7 \\
Total no recuperados & 8 & 14,8 \\
Curso crónico & 7 & 12,9 \\
Muertos & 1 & 1,8 \\
\hline
\end{tabular}

los casos en los predios se encontraban parasitados con mosca de los cuernos (Haematobia irritans).

El modelo de regresión logística múltiple que mejor se ajustó a los datos se presenta en el cuadro 4, el análisis de los residuales de Pearson indica que se cumple con el supuesto de linealidad con log (odds), además la distribución de los datos que predice el modelo no es diferente a la de los observados (test de bondad de ajuste de HosmerLemeshow $\mathrm{P}=0,72$ ). 
Cuadro 3. Características de los equinos participantes en el estudio, que se analizaron como potenciales factores de riesgo de APC, mediante regresión logística múltiple.

Characteristics of the equines that were analyzed as potential factors of APC risk, by means of multiple logistic regression.

\begin{tabular}{|c|c|c|c|c|}
\hline \multirow{2}{*}{$\begin{array}{c}\text { Características de los } \\
\text { equinos }\end{array}$} & $\begin{array}{c}\text { Enfermos } \\
(n=54)\end{array}$ & \multicolumn{2}{|c|}{$\begin{array}{c}\text { No enfer- } \\
\operatorname{mos}(n=85)\end{array}$} & $\begin{array}{c}\text { Total } \\
(\mathrm{n}=139)\end{array}$ \\
\hline & $\mathrm{N}^{\circ}$ & $\mathrm{N}^{\circ}$ & $\%^{*}$ & $\mathrm{~N}^{\circ}$ \\
\hline
\end{tabular}

$\begin{array}{lllllll}\text { Categoría animal } & & & & & & \\ \text { Potro } & 3 & 33,3 & 6 & 66,7 & 9 & 6,5 \\ \text { Yegua } & 37 & 41,6 & 52 & 58,4 & 89 & 64,0 \\ \text { Cría } & 3 & 27,3 & 8 & 72,7 & 11 & 7,9 \\ \text { Caballo } & 11 & 37,9 & 18 & 62,1 & 29 & 20,9\end{array}$

Estado corporal

$\begin{array}{lllllll}\text { Bueno } & 48 & 40,3 & 71 & 59,7 & 119 & 85,6 \\ \text { Regular } & 6 & 30,0 & 14 & 70,0 & 20 & 14,4\end{array}$

Tipo de estabulación

$\begin{array}{lllllll}\text { Permanente } & 3 & 42,9 & 4 & 57,1 & 7 & 5,0 \\ \text { Temporal } & 9 & 47,4 & 10 & 52,6 & 19 & 13,4 \\ \text { No estabula } & 42 & 37,2 & 71 & 62,8 & 113 & 81,3\end{array}$

Utilización del equino

$\begin{array}{lllllll}\text { Tiro } & 27 & 42,9 & 36 & 57,1 & 63 & 45,3 \\ \text { Paseo } & 13 & 39,4 & 20 & 60,6 & 33 & 23,7 \\ \quad \text { Deporte } & 4 & 17,4 & 19 & 82,6 & 23 & 16,5 \\ \quad \text { Sin amansar } & 7 & 41,2 & 10 & 58,8 & 17 & 12,2 \\ \text { Consumo de agua } & & & & & & \\ \quad \text { Canal } & 37 & 38,5 & 59 & 61,5 & 96 & 69,0 \\ \text { Pozo } & 17 & 39,5 & 26 & 60,5 & 43 & 30,9\end{array}$

Origen del caballo

$\begin{array}{lllllll}\text { Otro predio } & 6 & 60,0 & 4 & 40,0 & 10 & 7,2 \\ \quad \text { Feria } & 1 & 16,7 & 5 & 83,3 & 6 & 4,3 \\ \quad \text { Mismo predio } & 47 & 38,2 & 76 & 61,8 & 123 & 88,5 \\ \text { Tipo Criollo } & 45 & 48,4 & 48 & 51,6 & 93 & 66,9 \\ \text { Raza } & 9 & 19,6 & 37 & 80,4 & 46 & 33,1\end{array}$

Alimentación

$\begin{array}{lllllll}\text { Sólo pastoreo } & 39 & 37,1 & 66 & 62,9 & 105 & 75,5 \\ \text { Mixto } & 15 & 44,1 & 19 & 55,9 & 34 & 24,4\end{array}$

Uso antiparasitario

\begin{tabular}{lllllll} 
Anual & 35 & 52,2 & 32 & 47,8 & 67 & 48,2 \\
$\quad$ Ocasional & 12 & 24,0 & 38 & 76,0 & 50 & 36,0 \\
$\quad$ Nunca & 7 & 31,8 & 15 & 68,2 & 22 & 15,8 \\
$\begin{array}{l}\text { Predios con Mosca de } \\
\text { los cuernos }\end{array}$ & 31 & 100 & 0 & 0 & 31 & 100 \\
Edad: Media (SE) & $7,50(1,02)$ & $7,54(0,81)$ & $7,53(0,64)$ \\
\hline
\end{tabular}

*: Porcentajes de la fila, en relación a los enfermos y no enfermos.

\#: Porcentaje dentro de la columna, en relación a los niveles que tiene cada variable categórica.
Cuadro 4. Modelo final de regresión logística para la presentación de APC, frecuencia de los equinos en cada variable y su influencia en el modelo.

Final logistic regresion model for APC development, frequency of the equines in each variable and its influence in the model.

\begin{tabular}{lccccccc}
\hline & \multicolumn{3}{c}{ Equinos } & \multicolumn{5}{c}{$\begin{array}{c}\text { Parámetros modelo final } \\
\text { regresión logística múltiple }\end{array}$} \\
\hline \multicolumn{1}{c}{ Variable } & $\begin{array}{c}\text { Enfer- } \\
\text { mos }\end{array}$ & Total & $\begin{array}{c}\% \text { en- } \\
\text { fermos }\end{array}$ & OR & IC95\% OR & $\mathrm{p}$ \\
\hline $\begin{array}{l}\text { Tipo Criollo } \\
\text { Uso antiparasitario } \\
\text { anual }\end{array}$ & 45 & 93 & 48,4 & 6,179 & 2,433 & 15,693 & 0,000 \\
\hline
\end{tabular}

Los parámetros obtenidos señalan que los equinos de tipo criollo tuvieron 6,17 veces más probabilidad de enfermar que los demás de raza $(\mathrm{OR}=6,17$; IC $95 \%=2,43-15,69)$, y que la administración de antiparasitario en forma anual se asocia con aumento del riesgo de APC en 4,80 veces $(\mathrm{OR}=4,8 ;$ IC $95 \%=2,13-10,79)$; no se encontraron asociaciones estadísticas significativas $(\mathrm{P}>0,05)$ entre las variables analizadas.

El modelo planteado posee una capacidad predictiva general de 70,5\%, con una sensibilidad (Se) (capacidad de predecir un animal enfermo si es que realmente está enfermo) de 48,2\%, una mejor especificidad (Sp) (capacidad de predecir si un animal no está enfermo si realmente no tiene la enfermedad) $84,7 \%$, el área bajo la curva ROC es de 0,72 ; estos indicadores señalan que el modelo posee una moderada habilidad predictiva (Dohoo y col 2003).

\section{DISCUSION}

Las principales características de los equinos que se asociaron con el aumento del riesgo de desarrollar APC, en el brote ocurrido en la Provincia de Nuble son pertenecer al tipo criollo y ser desparasitados anualmente. Aunque los parámetros obtenidos no deben ser interpretados en sentido causal indican una fuerte asociación con la presentación de la enfermedad, lo que da un marco para analizar factores más específicos en estudios de casos y controles o de tipo prospectivo.

La condición de ser equino de tipo criollo es un factor significativo $(\mathrm{P}<0,01)$ para el aumento de la probabilidad de que un equino presente APC (OR $=6,17$; IC 95\% $=2,1$ 10,7). Desde los primeros reportes de brotes de APC se ha descrito que la raza (como predisposición intrínseca del animal) no es un factor de riesgo para contraer la enfermedad (Knight 1969); sin embargo, los animales de raza que poseen gran valor económico tienen diferentes condiciones de manejo, que los protegen de contraer la enfermedad (Doherr y col 1998b). Es así como las condiciones en que se mantienen los equinos criollos pudieran favorecer la presentación de APC. 
La administración de antiparasitario anual a los equinos también se asoció en forma significativa $(\mathrm{P}<0,01)$ al aumento del riesgo de contraer APC $(\mathrm{OR}=4,8 ; \mathrm{IC} 95 \%=2,1-10,7)$, lo que indica que las medidas de manejo sanitario de los equinos tienen alguna asociación con la susceptibilidad a esta enfermedad; esta relación puede ser también un factor de confusión, ya que existe la posibilidad de que además de estar relacionada con la variable respuesta (probabilidad de enfermar) se asocie a alguna otra variable no consultada en la encuesta en que sea más directa la estructura causal, se debe considerar también que exista un sesgo de información. Algunos autores (Knight 1969, Welsh 1990) señalan que las medidas sanitarias y de manejo no tienen incidencia en la presentación de la enfermedad; sin embargo, no se especifica qué medidas, ni el tipo y frecuencia de los fármacos utilizados. Existen antecedentes de que el uso de productos que actúen contra Hematobia irritans (mosca de los cuernos), principal portador de Corynebacterium pseudotuberculosis, podría tener un efecto positivo en disminuir el riesgo de enfermar (Miers y Ley 1980). A pesar de que el uso de antiparasitarios internos tiene importancia en la condición física general, una mejor condición corporal no se evidenció como factor de riesgo en el análisis.

Se ha reportado que trasladar animales desde una zona endémica a otra libre o en sentido inverso no influye en la presentación de la enfermedad (Aleman y col 1996); no obstante, Doherr y col (1999) sugieren que una posible razón para la ocurrencia de un brote podría darse si se introducen casos en una población de caballos altamente susceptibles, con gran número de equinos jóvenes, dando como resultado la diseminación de la enfermedad. En el presente brote no se tuvo evidencia para confirmarlo, ya que los animales que provenían de ferias ganaderas o de otros predios no se relacionaron con una mayor proporción de enfermos.

En otros brotes tampoco se encontró que el tipo de estabulación influenciara directamente la presentación de la enfermedad (Miers y Ley 1980, Aleman y col 1996); sin embargo, Doherr y col $\left(1998^{\mathrm{a}}\right.$ y $\left.1998^{\mathrm{b}}\right)$ indican que en caballos con estrecho contacto se incrementa significativamente el riesgo de enfermar principalmente cuando se encuentran casos de equinos con abscesos drenando que contaminan suelos y superficies; además, los insectos voladores tendrían un rol importante en la transmisión de la enfermedad, al ser muy abundantes en los lugares donde hay alta densidad de equinos y ser atraídos por las secreciones de los abscesos. El 100\% de los propietarios señaló que los animales estaban parasitados con Hematobia irritans al momento de presentarse la enfermedad, esta situación no permite incluirla en el modelo de regresión logística múltiple debido a que la ausencia de variabilidad le quita capacidad predictiva (Dohoo y col 2003); no obstante, no se puede descartar un probable rol de vector mecánico que puede cumplir este insecto en la diseminación de la enfermedad (Blood y Radostits 1992).
No se encontró evidencia de asociación significativa para la edad ni para la utilización de los equinos. En otros reportes se señalan riesgos diferenciales para las distintas edades (Aleman y col 1996, Doherr y col 1998ªb). También se indica que los equinos que desempeñan actividades deportivas, de caza, salto y trabajo tienen un riesgo significativo de contraer la enfermedad (Miers y Ley 1980, Doherr y col 1998 ab, Miers y Ley 1980).

El modelo descrito posee mejor Sp $(84,7 \%)$ que $\mathrm{Se}$ $(48,2 \%)$, por lo que es más probable que cometa menos errores al clasificar a los animales realmente sanos (Dohoo y col 2003), con una probabilidad total de clasificar correctamente de $70,50 \%$. Es necesario tomar en cuenta los diferentes sesgos que tienen los indicadores calculados, los que afectan la validez interna y externa de los resultados (Rothman 1998); en este caso pueden ser importantes, tanto los sesgos en los reportes de casos, como los de información, al ser poco precisa la información recogida por la encuesta debido al tipo de preguntas que fueron muy generales, o que corresponden a factores que varían en el tiempo y son difíciles de precisar, o que no relacionan condiciones inmediatamente anteriores al brote.

Los datos con los que se realizó el presente modelo provienen de un diseño de tipo transversal, por lo que sólo permite establecer asociaciones estadísticamente significativas, entre la probabilidad de ocurrencia de APC y las variables consideradas, pero no se pueden inferir relaciones causales entre ellas. Es posible que las asociaciones estadísticas encontradas sean parte de una red de confusores más amplia dentro de la estructura causal. Además, el diseño planteado no tuvo una estructura jerárquica, por lo que las características de los individuos examinados tuvieron una influencia uniforme en el modelo. Sería muy útil realizar un muestreo aleatorio de los predios e incluir este nivel en el modelo, de manera de que se considere la variabilidad entre los predios al calcular los parámetros.

En conclusión, el análisis multivariado de las características de los equinos, en relación a la probabilidad de desarrollar APC, en el brote ocurrido en el verano del año 2000, en la Provincia de Ñuble, VIII Región, indica como factores asociados al aumento del riesgo de haber enfermado el que los equinos sean de tipo criollo y utilizar antiparasitarios anualmente.

El aporte de esta investigación es que provee de antecedentes para el diseño de estudios de tipo casos y controles y luego de cohorte en que las asociaciones se puedan interpretar en sentido causal. Es recomendable que se estudien con más detalle las características intrínsecas y extrínsecas que poseen los equinos criollos, además de cuáles son los factores relacionados con la dosificación anual de antiparasitario que hacen que estas características se asocien fuertemente con la presentación de APC, de manera de aportar antecedentes para construir la estructura causal de la enfermedad en la VIII Región. 


\section{RESUMEN}

El absceso pectoral crónico (APC) es una enfermedad infecciosa de tipo estacional, que afecta a los equinos, causada por Corynebacterium pseudotuberculosis. Se realizó un estudio de tipo transversal en 31 predios de la Provincia de Ñuble que denunciaron casos de APC al Servicio Agrícola y Ganadero (SAG) desde noviembre de 1999 hasta abril de 2000. Se recolectó información individual y de manejo de todos los equinos de los predios que reportaron casos, mediante un cuestionario elaborado por el SAG. Los signos más frecuentes en los casos fueron: abscesos externos $(79,6 \%)$, edema $(100 \%)$, no se presentó necrosis, hubo decaimiento en el $61,1 \%$ de los casos y fiebre en el 31,5\%. A las 3 semanas se recuperó el $83,3 \%$ de los casos y el $12,9 \%$ siguió un curso crónico; mientras que la letalidad fue del 1,8\%. Mediante un análisis de regresión logística múltiple, utilizando Stata/SE V9 (StataCorp 2005), se encontró que las características: tipo criollo (OR $=6,17$; IC $95 \%=2,43-15,69)$ y uso de antiparasitario en forma anual $(\mathrm{OR}=4,8$; IC $95 \%=2,13-10,79)$ se asociaron con una mayor probabilidad de enfermar; en el brote descrito, el modelo planteado posee una capacidad predictiva general de 70,5\%. El aporte de esta investigación es proveer antecedentes para el diseño de estudios observacionales que se puedan interpretar en sentido causal. Es recomendable que se estudien con más detalle las características intrínsecas y extrínsecas que poseen los equinos criollos, además de cuáles son los factores relacionados con la dosificación anual de antiparasitario que hacen que estas características se asocien fuertemente con la presentación de APC, de manera de aportar antecedentes para construir la estructura causal de la enfermedad en la VIII Región.

\section{REFERENCIAS}

Aleman M, S Spier, W Wilson, M Doherr. 1996. Corynebacterium pseudotuberculosis infection in horses: 538 cases (1982-1993). $J$ Am Vet Med Assoc 209, 804-809.

Blood D, O Radostits. 1992. Medicina Veterinaria. Vol I, Ed. Interamericana, México.

Burrell D. 1981. Caseous lymphadenitis in goats. Aust Vet J 57, 105-110.

Colahan P, I Mayhew, A Merrit, J Moore. 1991. Equine medicine and surgery. $4^{\text {th }}$ ed. Vol I. American Veterinary Publications, California, USA.

Doherr M, T Carpenter, K Hanson, W Wilson, I Gardner. 1998ª . Risk factors associated with Corynebacterium pseudotuberculosis infection in California horses. Prev Vet Med 35, 229-239.
Doherr M, T Carpenter, W Wilson, I Gardner. 1998 . Application and evaluation of a mailed questionnaire for an epidemiologic study of Corynebacterium pseudotuberculosis infection in horses. Prev Vet Med 35, 241-253.

Doherr M, T Carpenter, W Wilson, I Gardner. 1999. Evaluation of temporal and spatial clustering of horses with Corynebacterium pseudotuberculosis infection. Am J Vet Res 60, 284-291.

Dohoo I, W Martin, H Stryhn. 2003. Veterinary Epidemiologic Research. AVC Inc., University Prince Edward Island, Canada.

Foley J, S Spier, J Mihalyi, N Drazenivich, C Leutenegger. 2004. Molecular epidemiologic features of Corynebacterium pseutotuberculosis isolated from horses. Am J Vet Res 65, 1734-1737.

Fraser C, J Bergeron, A Mays, S Aiello. 1993. El manual Merck de veterinaria. Merck \& Co, USA.

Hughes J, E Biberstein. 1959. Chronic equine abscesses associated with Corynebacterium pseudotuberculosis. J Am Vet Med Assoc 135, 559-562.

Knight H. 1969. Corynebacterial infections in the horse: problems of prevention. J Am Vet Med Assoc 155, 446-451.

Miers K, W Ley. 1980. Corynebacterium pseudotuberculosis infection in the horse: Study of 117 clinical cases and consideration of etiopathogenesis. J Am Vet Med Assoc 177, 250-253.

Perkins S, K Magdesian, W Thomas, S Spier. 2004. Pericarditis and pleuritis caused by Corynebacterium pseudotuberculosis in a horse. J Am Vet Med Assoc 7, 1133-1138.

Pinochet L. 1992. Linfoadenitis caseosa, un problema aún sin solución. Monografias Med Vet 14, 21-27.

Rothman K, S Greenland. 1998. Modern Epidemiology. $2^{\text {nd }}$ ed. LippincottRaven, USA.

Rumbaugh G, B Smith, G Carlson. 1978. Internal abdominal abscesses in the horse: a study of 25 cases. J Am Vet Med Assoc 172, 304-309.

Spiegel I, S White, J Foley, N Drazenovich, P Ihrke, V Affolter. 2006. A retrospective study of cutaneous equine sarcoidosis and its potential infectious aetological agents. Vet Dermatol 17, 51-62.

StataCorp 2005. Stata S/E Statistical Software: Release 9.0. College Station, TX: Stata Corporation.

Welsh R. 1990. Corynebacterium pseudotuberculosis in the horse. Equine practice 12, 7-16.

Wisecup W, D Blanchard, C Neil. 1964. Corynebacterium pseudotuberculosis associated with rapidly ocurring equine abscesses. J Am Vet Med Assoc 144, 152-154. 